\title{
MAXIMAL POLYNOMIALS AND THE ILIEFF-SENDOV CONJECTURE
}

\author{
MICHAEL J. MILLER
}

\begin{abstract}
In this paper, we consider those complex polynomials which have all their roots in the unit disk, one fixed root, and all the roots of their first derivatives as far as possible from a fixed point. We conjecture that any such polynomial has all the roots of its derivative on a circle centered at the fixed point, and as many of its own roots as possible on the unit circle. We prove a part of this conjecture, and use it to define an algorithm for constructing some of these polynomials. With this algorithm, we investigate the 1962 conjecture of Sendov and the 1969 conjecture of Goodman, Rahman and Ratti and (independently) Schmeisser, obtaining counterexamples of degrees $6,8,10$, and 12 for the latter.
\end{abstract}

\section{INTRODUCTION}

In this paper, $P$ will represent a polynomial with complex coefficients, and $D=\{z:|z| \leq 1\}$ will be the unit disk in the complex plane $\mathbb{C}$. In 1874 , Lucas proved [6]

Theorem 1.1. If all the roots of $P$ lie in $D$, then all the roots of $P^{\prime}$ also lie in $D$.

This is the best result possible, in the sense that, if all that is known about $P$ is that all its roots lie in $D$, then no proper subset of $D$ can be guaranteed to contain even one root of $P^{\prime}$ (as is shown by polynomials of the form $P(z)=$ $(z-a)^{n}$, since $P^{\prime}(z)=n(z-a)^{n-1}$ has roots only at $a$, which can lie anywhere in $D)$.

To locate the roots of $P^{\prime}$ more precisely, we need some additional information about $P$. We can get this by assuming not only that all roots of $P$ lie in $D$, but in addition that we know the precise location of one root $\beta$ of $P$. (Even this will allow us to locate at most one root of $P^{\prime}$, as is shown by polynomials of the form $P(z)=(z-\beta)(z-a)^{n}$.) Given this, our ultimate goal would be to

Received by the editors December 15, 1987 and, in revised form, November 3, 1988.

1980 Mathematics Subject Classification (1985 Revision). Primary 30C15.

Key words and phrases. Ilieff, Sendov, geometry of polynomials, roots of polynomials, maximal polynomials.

The contents of this paper are based on the author's doctoral dissertation, presented at the University of Rochester in July 1984. A sketch of the results was presented to the American Mathematical Society in January 1984. 
find a region of smallest area, depending on at most $\beta$ and the degree of $P$, which would be guaranteed to contain at least one root of $P^{\prime}$.

While this goal is very far from being achieved, there are some conjectures which address questions of this type. One of them, due to Sendov in 1962 but erroneously attributed to Ilieff by Hayman in 1967 [5, p. 25], is

Conjecture 1.2. If all the roots of $P$ lie in $D$ and $\beta$ is a root of $P$ then the disk $\{z:|z-\beta| \leq 1\}$ contains at least one root of $P^{\prime}$.

A second, stronger conjecture put forth in 1969 by Goodman, Rahman, and Ratti [4] and independently by Schmeisser [12] is

Conjecture 1.3. If all the roots of $P$ lie in $D$ and $\beta$ is a root of $P$ then the disk $\{z:|z-\beta / 2| \leq 1-|\beta| / 2\}$ contains at least one root of $P^{\prime}$.

That this implies Conjecture 1.2 comes from the observation that the disk $\{z:|z-\beta / 2| \leq 1-|\beta| / 2\}$ is contained in the disk $\{z:|z-\beta| \leq 1\}$.

In still another attempt to shed light on Conjecture 1.2, Phelps and Rodriguez [10] have made the

Definitions 1.4. (1) $S_{n}$ is the set of all polynomials of degree $n$ which have all their roots in $D$.

(2) $I(P)=\max \left\{\min \left\{|z-w|: P^{\prime}(z)=0\right\}: P(w)=0\right\}$.

(3) $I\left(S_{n}\right)=\sup \left\{I(P): P \in S_{n}\right\}$.

(4) $P \in S_{n}$ is an extremal polynomial for Conjecture 1.2 if $I(P)=I\left(S_{n}\right)$.

Notice that if $P \in S_{n}$ and $\beta$ is a root of $P$ then there is at least one root of $P^{\prime}$ in $\{z:|z-\beta| \leq I(P)\}$. Phelps and Rodriguez make the

Conjecture 1.5. If $P \in S_{n}$ is an extremal polynomial for Conjecture 1.2 then $P(z)=z^{n}-a$ for some $a$ with $|a|=1$.

Note that for $P(z)=z^{n}-a, I(P)=\sqrt[n]{|a|}$. Thus Conjecture 1.5 implies that $I\left(S_{n}\right)=1$, which implies Conjecture 1.2.

Some twenty papers have been published on these conjectures, and a number of partial results have been obtained. In particular, Conjecture 1.3 is known to be true for all polynomials of degree at most 5 [3], and when $|\beta|=1[4,12]$. Conjecture 1.2 is also true in these cases (since it is implied by Conjecture 1.3). In addition, Conjecture 1.2 is known to be true when the convex hull $H$ of the roots of $P$ is a line segment or a triangle [13], when $H$ has all its vertices on the unit circle [12], when at least $2 / 3$ of the coefficients of $P$ are 0 [13], and for a number of other special cases (see [8, 13]). As for Conjecture 1.5, Phelps and Rodriguez have shown [10] that at least two roots of an extremal polynomial must lie on the unit circle.

Notice that Conjectures 1.2 and 1.3 are really estimates of the maximum distance possible between a fixed point ( $\beta$ in Conjecture 1.2 and $\beta / 2$ in Conjecture 1.3) and the nearest root of $P^{\prime}$. In $\S 2$, we consider worst possible cases for these conjectures, in the sense that we consider polynomials which have 
all their roots in the unit disk, one fixed root, and all the roots of their first derivatives as far as possible from a fixed point. We make a conjecture about the location of the roots of such a polynomial and the roots of its derivative, and we prove that specified rational functions of these roots must satisfy a certain linear condition. In $\S 3$, we use this linear condition to prove a part of our conjecture. In $\S 4$, we use the conjecture to define an algorithm for constructing some of these polynomials. With this algorithm, we investigate the 1962 conjecture of Sendov and the 1969 conjecture of Goodman, Rahman and Ratti and (independently) Schmeisser, obtaining inconclusive results for the former and counterexamples of degrees $6,8,10$, and 12 for the latter.

\section{MaXimality AND 2-MAXIMALITY}

We begin by making

Definitions 2.1. (1) $S(n, \beta)$ is the set of all polynomials of degree $n$ which have all their roots in $D$ and at least one root at $\beta$.

(2) $|P|_{\alpha}=\min \left\{|\zeta-\alpha|: P^{\prime}(\zeta)=0\right\}$.

Thus $|P|_{\alpha}$ is the distance from $\alpha$ to the closest root of $P^{\prime}$. In this notation, Conjecture 1.2 is that for all $P \in S(n, \beta),|P|_{\beta} \leq 1$, and Conjecture 1.3 is that for all $P \in S(n, \beta),|P|_{\beta / 2} \leq 1-|\beta| / 2$.

Finally, we make

Definition 2.2. $P \in S(n, \beta)$ is maximal with respect to $\alpha$ if $|P|_{\alpha} \geq|Q|_{\alpha}$ for all $Q \in S(n, \beta)$.

Thus, $P \in S(n, \beta)$ is maximal with respect to $\alpha$ if the roots of its derivative are as far as possible away from $\alpha$. These maximal polynomials will be our "worst possible cases", where we choose $\alpha=\beta$ for Conjecture 1.2 and $\alpha=\beta / 2$ for Conjecture 1.3.

Proposition 2.3. For each $n, \alpha$, and $\beta$ there is a $P \in S(n, \beta)$ which is maximal with respect to $\alpha$.

Proof. Let $P(z)=(z-\beta) Q(z)$. Then $|P|_{\alpha}$ is continuous in the roots of $P^{\prime}$, which are continuous in the coefficients of $P^{\prime}$, which are continuous in the coefficients of $Q$, which are continuous in the roots of $Q$ (being elementary symmetric functions of those roots), which are contained in the compast set $D$, so $|P|_{\alpha}$ achieves its supremum on $D$.

Besides the obvious relevance of maximal polynomials to Conjectures 1.2 and 1.3 , we have

Proposition 2.4. If $P \in S_{n}$ is an extremal polynomial for Conjecture 1.2, then there is a root $\beta$ of $P$ so that $P \in S(n, \beta)$ is maximal with respect to $\beta$.

Proof. Note that $I(P)=\max \left\{|P|_{w}: P(w)=0\right\}$. Suppose $P \in S_{n}$ is extremal, and let $\beta$ be a root of $P$ so that $I(P)=|P|_{\beta}$. If there were a $Q \in S(n, \beta)$ 
so that $|Q|_{\beta}>|P|_{\beta}$, then $Q \in S_{n}$ and $I(Q) \geq|Q|_{\beta}>|P|_{\beta}=I(P)$ so $P$. is not extremal, which is a contradiction. Thus $P \in S(n, \beta)$ is maximal with respect to $\beta$.

Thus, characterizing maximal polynomials will provide a great deal of information about Conjectures 1.2, 1.3, and 1.5. Based on a number of computer searches for these maximal polynomials, the author proposes

Conjecture 2.5. Suppose $P \in S(n, \beta)$ is maximal with respect to $\alpha$. Then

(1) all roots of $P^{\prime}$ lie on the circle $\left\{z:|z-\alpha|=|P|_{\alpha}\right\}$ and, given this, as many roots of $P$ as possible lie on the circle $\{z:|z|=1\}$;

(2) if $\alpha$ and $\beta$ are real and $P$ is monic then $P$ is a real polynomial.

By "as many roots of $P$ as possible", we mean that $P$ has $l$ roots on the unit circle, where $l$ is the largest integer such that there exists a polynomial $R \in S(n, \beta)$ with all roots of $R^{\prime}$ on a circle centered at $\alpha$ and $l$ roots of $R$ on the unit circle.

In attempting to prove Conjecture 2.5, we will have occasion to use the logarithmic derivative $P^{\prime} / P$. Unfortunately for our purposes, $P^{\prime} / P$ does not have as zeros those roots of $P^{\prime}$ which come from multiple roots of $P$. To get around this, we begin by defining a slightly different norm. Note that it would not suffice to simply define the norm to be the distance between $\alpha$ and the closest root of $P^{\prime} / P$ (as this would allow maximal polynomials to have multiple roots at $\beta)$.

Instead, we make

Definitions 2.6. (1) For $P(z)=(z-\beta) \prod_{j=1}^{k}\left(z-z_{j}\right)^{m_{j}}$ with distinct $z_{j}$,

$$
\|P\|_{\alpha}=\min \left\{|\zeta-\alpha|: \zeta \text { is a root of } P^{\prime}(z) / \prod_{j=1}^{k}\left(z-z_{j}\right)^{m_{j}-1}\right\} \text {. }
$$

(2) $P \in S(n, \beta)$ is 2-maximal with respect to $\alpha$ if $\|P\|_{\alpha} \geq\|Q\|_{\alpha}$ for all $Q \in S(n, \beta)$.

Notice that $\|P\|_{\alpha} \geq|P|_{\alpha}$ (since we take the minimum over a smaller set), with equality if $P$ has no multiple roots.

We must be more careful in asserting the existence of 2-maximal polynomials, as $\|P\|_{\alpha}$ is not continuous in the roots of $P$ (if the root $\zeta$ of $P^{\prime}(z) / \prod_{j=1}^{k}\left(z-z_{j}\right)^{m_{j}-1}$ closest to $\alpha$ comes from two roots of $P$ which coalesce, then $\zeta$ will become a multiple root of $P$ and hence will not appear in the minimum). $\|P\|_{\alpha}$ is, however, upper semicontinuous in the roots of $P^{\prime}$, and with this alteration to the proof of Proposition 2.3, we obtain

Proposition 2.7. For each $n, \alpha$, and $\beta$ there is a polynomial $P \in S(n, \beta)$ which is 2-maximal with respect to $\alpha$.

Now, it may well happen that $\beta$ is a multiple root of a 2 -maximal polynomial. As an example, $P(z)=(z-1)^{n} \in S(n, 1)$ is 2 -maximal with respect to 
-1 (since $\left.P^{\prime}(z) / \prod_{j=1}^{k}\left(z-z_{j}\right)^{m_{j}-1}=n(z-1)\right)$. We would like to eliminate this possibility, as it becomes irksome in our later arguments. As such, we need to determine when we can assume that a 2-maximal polynomial in $S(n, \beta)$ will not have $\beta$ as a multiple root. We achieve this via

Proposition 2.8. If $|\beta|<1$ and $P \in S(n, \beta)$ is 2-maximal with respect to $\alpha$, then $\|P\|_{\alpha}>|\alpha-\beta|$.

Proof. Let $K$ be a disk contained in the unit disk such that the point in $K$ closest to $\alpha$ is $\beta$, and let $Q$ be a polynomial with simple roots (including $\beta$ ) in $K$. By Theorem 1.1, all the roots of $Q^{\prime}$ lie in $K$. Since $\beta$ is not a root of $Q^{\prime}$, all roots of $Q^{\prime}$ are further from $\alpha$ than $\beta$, so $\|Q\|_{\alpha}>|\alpha-\beta|$. Since $P$ is 2-maximal, $\|P\|_{\alpha} \geq\|Q\|_{\alpha}$, and the result follows.

Corollary 2.9. If $|\beta|<1$ and $P \in S(n, \beta)$ is 2-maximal with respect to $\alpha$ then $P^{\prime}(\beta) \neq 0$.

Proof. Suppose $P(z)=(z-\beta) \prod_{j=1}^{k}\left(z-z_{j}\right)^{m_{j}}$ with distinct $z_{j}$. If $P^{\prime}(\beta)=0$ then $\beta$ is a multiple root of $P$, hence a root of $P^{\prime}(z) / \prod_{j=1}^{k}\left(z-z_{j}\right)^{m_{j}-1}$, so $\|P\|_{\alpha} \leq|\alpha-\beta|$, which contradicts Proposition 2.8.

It would seem useful at this stage to define a local version of 2-maximality. We shall not, however, as it appears that proving an analog of Corollary 2.9 for local 2-maximality would be quite difficult. Nevertheless, such local ideas will be an underlying motivation for our arguments.

Definition 2.10. For $P(z)=(z-\beta) \prod_{j=1}^{k}\left(z-z_{j}\right)^{m_{j}} \in S(n, \beta)$ with distinct $z_{j}$, a perturbation $P_{t}$ of $P$ is a family of polynomials

$$
P_{t}(z)=(z-\beta) \prod_{j=1}^{k}\left(z-z_{j}(t)\right)^{m_{j}}
$$

where each $z_{j}:[0,1] \rightarrow D$ is differentiable and $z_{j}(0)=z_{j}$.

Note that $P_{0}(z)=P(z)$. We then have the trivial

Proposition 2.11. If $P \in S(n, \beta)$ is 2-maximal with respect to $\alpha$ then there is no perturbation $P_{t}$ of $P$ so that $\left\|P_{t}\right\|_{\alpha}$ is a strictly increasing function of $t$ in a neighborhood of 0 .

From this point on, let us assume the

Hypotheses 2.12. (1) $|\beta|<1$.

(2) $P \in S(n, \beta)$ is 2 -maximal with respect to $\alpha$.

We make

Definition 2.13. The critical circle is the circle $\left\{z:|z-\alpha|=\|P\|_{\alpha}\right\}$; 
and we adopt the

Notation 2.14. (1) $P(z)=(z-\beta) \prod_{j=1}^{k}\left(z-z_{j}\right)^{m_{j}}$, with $\beta, z_{1}, \ldots, z_{k}$ distinct.

(2) $z_{1}, \ldots, z_{s}$ lie on $\{z:|z|=1\}$ and $z_{s+1}, \ldots, z_{k}$ lie in $\{z:|z|<1\}$.

(3) The roots of $P^{\prime}(z) / \prod_{j=1}^{k}\left(z-z_{j}\right)^{m_{j}-1}$ are $\zeta_{1}, \ldots, \zeta_{k}$.

(4) $\zeta_{1}, \ldots, \zeta_{r}$ lie on the critical circle and $\zeta_{r+1}, \ldots, \zeta_{k}$ lie in $\{z:|z-\alpha|>$ $\left.\|P\|_{\alpha}\right\}$.

To make use of Proposition 2.11 we need to know how $\left\|P_{t}\right\|_{\alpha}$ depends on the roots of $P_{t}$, so we need to know how the roots of $P_{t}^{\prime}$ depend on the roots of $P_{t}$. We achieve this via

Lemma 2.15. If $\zeta_{0}$ is a simple root of $P^{\prime}(z) / \prod_{j=1}^{k}\left(z-z_{j}\right)^{m_{j}-1}$ and $P_{t}$ is a perturbation of $P$ then $P_{t}^{\prime}(\zeta)=0$ defines $\zeta$ implicitly as a differentiable function of $t$ in a neighborhood of $t=0, \zeta=\zeta_{0}$. Furthermore,

$$
\zeta^{\prime}(0)=A\left(\zeta_{0}\right)\left(\zeta_{0}-\alpha\right) \sum_{j=1}^{k} \frac{m_{j}}{\left(\zeta_{0}-z_{j}\right)^{2}} z_{j}^{\prime}(0),
$$

where $A\left(\zeta_{0}\right)$ is defined by

\section{Definition 2.16.}

$$
A\left(\zeta_{0}\right)=\frac{1}{\zeta_{0}-\alpha}\left[\frac{1}{\left(\zeta_{0}-\beta\right)^{2}}+\sum_{j=1}^{k} \frac{m_{j}}{\left(\zeta_{0}-z_{j}\right)^{2}}\right]^{-1}=\frac{-1}{\zeta_{0}-\alpha} \frac{P\left(\zeta_{0}\right)}{P^{\prime \prime}\left(\zeta_{0}\right)}
$$

Proof of Lemma 2.15. Note first that $P\left(\zeta_{0}\right) \neq 0$, since no roots are shared by $P(z)$ and $P^{\prime}(z) / \prod_{j=1}^{k}\left(z-z_{j}\right)^{m_{j}-1}$. In addition, $P^{\prime \prime}\left(\zeta_{0}\right) \neq 0$ since $\zeta_{0}$ is a simple root of $P^{\prime}$. Now

$$
\frac{P^{\prime}(\zeta)}{P(\zeta)}=\frac{1}{\zeta-\beta}+\sum_{j=1}^{k} \frac{m_{j}}{\zeta-z_{j}}
$$

so

$$
0 \neq \frac{P^{\prime \prime}\left(\zeta_{0}\right)}{P\left(\zeta_{0}\right)}=\left.\left(\frac{P^{\prime}(\zeta)}{P(\zeta)}\right)^{\prime}\right|_{\zeta=\zeta_{0}}=-\left[\frac{1}{\left(\zeta_{0}-\beta\right)^{2}}+\sum_{j=1}^{k} \frac{m_{j}}{\left(\zeta_{0}-z_{j}\right)^{2}}\right]
$$

Also, $\zeta_{0} \neq \alpha$ (else $\|P\|_{\alpha}=0$, which is disallowed by Proposition 2.8 ), so the expression for $A\left(\zeta_{0}\right)$ makes sense.

Let $F(\zeta, t)=P_{t}^{\prime}(\zeta)$. Then $F_{\zeta}\left(\zeta_{0}, 0\right)=P^{\prime \prime}\left(\zeta_{0}\right) \neq 0$, so $F(\zeta, t)=0$ defines $\zeta$ implicitly as a differentiable function of $t$ in a neighborhood of $\left(\zeta_{0}, 0\right)$. 
Further,

$$
\begin{aligned}
F_{t}\left(\zeta_{0}, 0\right) & =\left.\frac{d}{d t} P_{t}^{\prime}\left(\zeta_{0}\right)\right|_{t=0}=\left.\frac{d}{d t}\left(\frac{P_{t}^{\prime}\left(\zeta_{0}\right)}{P_{t}\left(\zeta_{0}\right)} P_{t}\left(\zeta_{0}\right)\right)\right|_{t=0} \\
& =\left.\frac{P^{\prime}\left(\zeta_{0}\right)}{P\left(\zeta_{0}\right)} \frac{d}{d t} P_{t}\left(\zeta_{0}\right)\right|_{t=0}+\left.P\left(\zeta_{0}\right) \frac{d}{d t} \frac{P_{t}^{\prime}\left(\zeta_{0}\right)}{P_{t}\left(\zeta_{0}\right)}\right|_{t=0} \\
& =0+\left.P\left(\zeta_{0}\right) \frac{d}{d t}\left[\frac{1}{\zeta_{0}-\beta}+\sum_{j=1}^{k} \frac{m_{j}}{\zeta_{0}-z_{j}(t)}\right]\right|_{t=0} \\
& =P\left(\zeta_{0}\right) \sum_{j=1}^{k} \frac{m_{j}}{\left(\zeta_{0}-z_{j}\right)^{2}} z_{j}^{\prime}(0)
\end{aligned}
$$

so

$$
\begin{aligned}
\zeta^{\prime}(0) & =-\frac{F_{t}\left(\zeta_{0}, 0\right)}{F_{\zeta}\left(\zeta_{0}, 0\right)}=-\frac{P\left(\zeta_{0}\right) \sum_{j=1}^{k} m_{j} z_{j}^{\prime}(0) /\left(\zeta_{0}-z_{j}\right)^{2}}{P^{\prime \prime}\left(\zeta_{0}\right)} \\
& =A\left(\zeta_{0}\right)\left(\zeta_{0}-\alpha\right) \sum_{j=1}^{k} \frac{m_{j}}{\left(\zeta_{0}-z_{j}\right)^{2}} z_{j}^{\prime}(0) . \quad \square
\end{aligned}
$$

Proposition 2.11 implies that there is no perturbation $P_{t}$ of $P$ such that $\left|z_{j}(t)\right|$ is decreasing for $j=1, \ldots, s$ and $\left|\zeta_{i}(t)-\alpha\right|$ is strictly increasing for $i=1, \ldots, r$. We linearize these conditions via

Lemma 2.17. Suppose $z:[0,1] \rightarrow \mathbb{C}$ is differentiable and $\alpha \neq z(0)$. If

$$
\operatorname{Re}\left(z^{\prime}(0) /(z(0)-\alpha)\right)>0 \quad(\text { resp. }<0)
$$

then $|z(t)-\alpha|$ is a strictly increasing (resp. decreasing) function of $t$ in a neighborhood of 0 .

Proof. Write $z(t)-\alpha=\rho(t) e^{i \varphi(t)}$. Then

$$
\frac{z^{\prime}(t)}{z(t)-\alpha}=\frac{\rho^{\prime}(t)}{\rho(t)}+i \varphi^{\prime}(t),
$$

so $\operatorname{Re}\left(z^{\prime}(0) /(z(0)-\alpha)\right)=\rho^{\prime}(0) / \rho(0)$ and the result follows.

Thus, there is no perturbation $P_{t}$ of $P$ such that $\operatorname{Re}\left(z_{j}^{\prime}(0) / z_{j}\right)<0$ for $j=1, \ldots, s$ and $\operatorname{Re}\left(\zeta_{i}^{\prime}(0) /\left(\zeta_{i}-\alpha\right)\right)>0$ for $i=1, \ldots, r$. Now if $\zeta_{i}$ is a simple root of $P^{\prime}(z) / \prod_{j=1}^{k}\left(z-z_{j}\right)^{m_{j}-1}$,

$$
\begin{aligned}
\zeta_{i}^{\prime}(0) & =A\left(\zeta_{i}\right)\left(\zeta_{i}-\alpha\right) \sum_{j=1}^{k} \frac{m_{j}}{\left(\zeta_{i}-z_{j}\right)^{2}} z_{j}^{\prime}(0) \quad \text { by Lemma } 2.15 \\
& =A\left(\zeta_{i}\right)\left(\zeta_{i}-\alpha\right)\left[\sum_{j=1}^{s} \frac{z_{j}}{\left(\zeta_{i}-z_{j}\right)^{2}} \frac{m_{j} z_{j}^{\prime}(0)}{z_{j}}+\sum_{j=s+1}^{k} \frac{1}{\left(\zeta_{i}-z_{j}\right)^{2}} m_{j} z_{j}^{\prime}(0)\right],
\end{aligned}
$$


so the second conditions translate to

$$
\operatorname{Re}\left[\sum_{j=1}^{s} \frac{A\left(\zeta_{i}\right) z_{j}}{\left(\zeta_{i}-z_{j}\right)^{2}} \frac{m_{j} z_{j}^{\prime}(0)}{z_{j}}+\sum_{j=s+1}^{k} \frac{A\left(\zeta_{i}\right)}{\left(\zeta_{i}-z_{j}\right)^{2}} m_{j} z_{j}^{\prime}(0)\right]>0
$$

for $i=1, \ldots, r$. Thus,

\section{System 2.18.}

$\operatorname{Re}\left(\begin{array}{cccccc}\frac{A\left(\zeta_{1}\right) z_{1}}{\left(\zeta_{1}-z_{1}\right)^{2}} & \cdots & \frac{A\left(\zeta_{1}\right) z_{s}}{\left(\zeta_{1}-z_{s}\right)^{2}} & \frac{A\left(\zeta_{1}\right)}{\left(\zeta_{1}-z_{s+1}\right)^{2}} & \cdots & \frac{A\left(\zeta_{1}\right)}{\left(\zeta_{1}-z_{k}\right)^{2}} \\ \vdots & & \vdots & \vdots & & \vdots \\ \frac{A\left(\zeta_{r}\right) z_{1}}{\left(\zeta_{r}-z_{1}\right)^{2}} & \cdots & \frac{A\left(\zeta_{r}\right) z_{s}}{\left(\zeta_{r}-z_{s}\right)^{2}} & \frac{A\left(\zeta_{r}\right)}{\left(\zeta_{r}-z_{s+1}\right)^{2}} & \cdots & \frac{A\left(\zeta_{r}\right)}{\left(\zeta_{r}-z_{k}\right)^{2}} \\ -1 & 0 \cdots & 0 & 0 & \cdots & 0 \\ 0 & & \vdots & \vdots & & \vdots \\ \vdots & & 0 & & & \\ 0 & \cdots 0 & -1 & 0 & \cdots & 0\end{array}\right)\left(\begin{array}{c}m_{1} z_{1}^{\prime}(0) / z_{1} \\ \vdots \\ m_{s} z_{s}^{\prime}(0) / z_{s} \\ m_{s+1} z_{s+1}^{\prime}(0) \\ \vdots \\ m_{k} z_{k}^{\prime}(0)\end{array}\right)>0$

can have no solution. We ask now what this implies about the coefficient matrix of this system.

Definition 2.19. An $m \times n$ matrix $A=\left(a_{i j}\right)$ is positively singular if there are numbers $\beta_{1}, \ldots, \beta_{m} \geq 0$, not all 0 , so that $\sum_{i=1}^{m} \beta_{i} a_{i j}=0$ for $j=1, \ldots, n$.

Thus, a matrix is positively singular if positive multiples of some of its rows sum to the zero vector.

A minor adaption of a theorem of J. Farkas [2, p. 5] leads to the result that, for $A$ a real $m \times n$ matrix and $\vec{x}$ an $n$-vector of real unknowns, the system $A \vec{x}>\overrightarrow{0}$ has no solutions if and only if $A$ is positively singular. We generalize this via the

Lemma 2.20. For $A$ a complex $m \times n$ matrix and $\vec{z}$ an n-vector of complex unknowns, the system $\operatorname{Re}(A \vec{z})>\overrightarrow{0}$ has no solutions if and only if $A$ is positively singular.

Proof. Break the system $\operatorname{Re}(A \vec{z})>\overrightarrow{0}$ into a system with coefficients the real and imaginary parts of the entries of $A$ and with variables the real and imaginary parts of the entries of $\vec{z}$, and apply the Farkas result to this new system.

Thus, we have proved the main result of this section:

Theorem 2.21. If $P^{\prime}(z) / \prod_{j=1}^{k}\left(z-z_{j}\right)^{m_{j}-1}$ has no multiple roots on the critical 
circle, then the matrix

$$
\left(\begin{array}{cccccc}
\frac{A\left(\zeta_{1}\right) z_{1}}{\left(\zeta_{1}-z_{1}\right)^{2}} & \cdots & \frac{A\left(\zeta_{1}\right) z_{s}}{\left(\zeta_{1}-z_{s}\right)^{2}} & \frac{A\left(\zeta_{1}\right) z_{s}}{\left(\zeta_{1}-z_{s+1}\right)^{2}} & \cdots & \frac{A\left(\zeta_{1}\right)}{\left(\zeta_{1}-z_{k}\right)^{2}} \\
\vdots & & \vdots & \vdots & & \vdots \\
\frac{A\left(\zeta_{r}\right) z_{1}}{\left(\zeta_{r}-z_{1}\right)^{2}} & \cdots & \frac{A\left(\zeta_{r}\right) z_{s}}{\left(\zeta_{r}-z_{s}\right)^{2}} & \frac{A\left(\zeta_{r}\right) z_{s}}{\left(\zeta_{r}-z_{s+1}\right)^{2}} & \cdots & \frac{A\left(\zeta_{r}\right)}{\left(\zeta_{r}-z_{k}\right)^{2}} \\
-1 & 0 \cdots & 0 & 0 & \cdots & 0 \\
0 & & \vdots & \vdots & & \vdots \\
\vdots & & 0 & & & \\
0 & \cdots 0 & -1 & 0 & \cdots & 0
\end{array}\right)
$$

is positively singular.

\section{IMPLicATIONS OF THEOREM 2.21}

A major step in verifying Conjecture 2.5 would be to prove that $r=k$. While we will not be able to do this, we will show, under appropriate hypotheses, that $r \geq 2$ and that $r \geq(k+2) / 6$.

We begin with the following well-known result [11, p. 43].

Lemma 3.1. If $h$ is analytic and nonzero on $C=\{z:|z|=1\}$ and

$$
\operatorname{Re}\left(z h^{\prime}(z) / h(z)\right)<0
$$

on $C$ then $\arg h\left(e^{i t}\right)$ is a strictly decreasing function of $t$.

We use this to verify

Lemma 3.2. For any $\zeta$ with $|\zeta|<1$, the image of $C=\{z:|z|=1\}$ under the mapping $z \mapsto z /(\zeta-z)^{2}$ intersects each ray from the origin exactly once.

Proof. Let $h(z)=z /(\zeta-z)^{2}$. Then $h$ is analytic and nonzero on $C$. Further,

$$
\frac{z h^{\prime}(z)}{h(z)}=z\left(\frac{1}{z}+\frac{2}{\zeta-z}\right)=\frac{\zeta+z}{\zeta-z},
$$

so $\operatorname{Re}\left(z h^{\prime}(z) / h(z)\right)<0$ on $C$ (since $z \mapsto(\zeta+z) /(\zeta-z)$ maps $\{z:|z| \geq|\zeta|\}$ to $\{z: \operatorname{Re} z \leq 0\})$. Thus by Lemma 3.1, $\arg h\left(e^{i t}\right)$ is a strictly decreasing function of $t$. Now, the net change in $\arg h\left(e^{i t}\right)$ as $t$ increases from 0 to $2 \pi$ is $2 \pi$ (the number of roots of $h$ inside $C-$ the number of poles of $h$ inside $C$ ) $=2 \pi(1-2)^{\circ}=-2 \pi$. Thus, as $t$ increases from 0 to $2 \pi, \arg h\left(e^{i t}\right)$ strictly decreases by exactly $2 \pi$.

Now we are ready to prove the first main result of this section.

Theorem 3.3. If $|\beta|<1, P \in S(n, \beta)$ is 2-maximal with respect to $\alpha$ and $k \geq 2$ then $r \geq 2$, i.e. $P^{\prime}(z) / \prod_{j=1}^{k}\left(z-z_{j}\right)^{m_{j}-1}$ has at least 2 roots on the critical circle. 
Proof. If $P^{\prime}(z) / \prod_{j=1}^{k}\left(z-z_{j}\right)^{m_{j}-1}$ has only the simple root $\zeta_{1}$ on the critical circle, then by Theorem 2.21 the matrix

$$
\left(\begin{array}{cccccc}
\frac{A\left(\zeta_{1}\right) z_{1}}{\left(\zeta_{1}-z_{1}\right)^{2}} & \cdots & \frac{A\left(\zeta_{1}\right) z_{s}}{\left(\zeta_{1}-z_{s}\right)^{2}} & \frac{A\left(\zeta_{1}\right)}{\left(\zeta_{1}-z_{s+1}\right)^{2}} & \cdots & \frac{A\left(\zeta_{1}\right)}{\left(\zeta_{1}-z_{k}\right)^{2}} \\
-1 & 0 \cdots & 0 & 0 & \cdots & 0 \\
0 & \ddots & \vdots & \vdots & & \vdots \\
\vdots & & 0 & & & \\
0 & \cdots 0 & -1 & 0 & \cdots & 0
\end{array}\right)
$$

is positively singular. Thus for $j=1, \ldots, s$ each $A\left(\zeta_{1}\right) z_{j} /\left(\zeta_{1}-z_{j}\right)^{2}>0$ so each $z_{j} /\left(\zeta_{1}-z_{j}\right)^{2}$ lies on the same ray through the origin and on the image of $\{z:|z|=1\}$ under the mapping $z \mapsto z /\left(\zeta_{1}-z\right)^{2}$. By Lemma 3.2 this can happen at most once, so $s \leq 1$. If $k>s$ then $A\left(\zeta_{1}\right) /\left(\zeta_{1}-z_{s+1}\right)^{2}=0$, which is impossible. Thus $k=s \leq 1$, which is a contradiction.

We now make the

Definition 3.4. The maximal degree of a rational function $h(z)=P(z) / Q(z)$ is $\max \{\operatorname{deg} P, \operatorname{deg} Q\}$.

We then prove

Lemma 3.5. Let $h(z)$ be a rational function of maximal degree $N$ and let $C$ be a circle (or a line) in $\mathbb{C}$. Then either $h$ is real on all of $C$ or $h$ is real on at most $2 N$ points of $C$.

Proof. Write $h(z)=P(z) / Q(z)$ with $Q$ monic and $N=\max \{\operatorname{deg} P, \operatorname{deg} Q\}$.

Case I: $C=\mathbb{R}$. Suppose that $x_{1}, \ldots, x_{2 N+1}$ are distinct points in $\mathbb{R}$ so that $y_{i}=h\left(x_{i}\right) \in \mathbb{R}$ for $i=1, \ldots, 2 N+1$. Let $\widehat{P}$ (resp. $\widehat{Q}$ ) be the polynomial whose coefficients are the real parts of the coefficients of $P$ (resp. $Q$ ). Then $\widehat{P}\left(x_{i}\right) Q\left(x_{i}\right)=y_{i} \widehat{Q}\left(x_{i}\right) Q\left(x_{i}\right)=\widehat{Q}\left(x_{i}\right) P\left(x_{i}\right)$, so $\widehat{P} Q-\widehat{Q} P$ is a polynomial of degree $\leq 2 N$ which vanishes at $2 N+1$ distinct points, so $\widehat{P} Q-\widehat{Q} P \equiv 0$. Then $h(x)=P(x) / Q(x)=\widehat{P}(x) / \widehat{Q}(x)$ is real on all of $\mathbb{R}$.

Case II: $C \neq \mathbb{R}$. Let $\mu(z)=(a z+b) /(c z+d)$ map $\mathbb{R}$ to $C$. Then $h \circ \mu(z)=$ $P((a z+b) /(c z+d)) / Q((a z+b) /(c z+d))$ is a rational function of maximal degree $N$ (after multiplying numerator and denominator by $(c z+d)^{N}$ ), so by Case I $h \circ \mu$ is real on all of $\mathbb{R}$ or real on at most $2 N$ points of $\mathbb{R}$. Thus $h$ is real on all of $C$ or real on at most $2 N$ points of $C$.

Note that Lemma 3.5 is the best we can expect, as is shown by $h(z)=1 / z^{N}$, which is real $2 N$ times on $\{z:|z|=1\}$.

We now show

Lemma 3.6. If $S$ is a closed subset of $\mathbb{C} \cup\{\infty\}$ with nonvoid interior, then a nonconstant analytic function $f$ on $S$ cannot be real on all of $\partial S$. 
Proof. Let $T$ be the image of $S$ under $f$. T is compact (since $f$ is analytic) and $T \nsubseteq \mathbb{R}$ (since $T$ has nonvoid interior), so there is a $z_{0} \in S$ such that $f\left(z_{0}\right)$ is of (positive) maximal distance from $\mathbb{R}$. But then $f\left(z_{0}\right) \in \partial T$ so $z_{0} \in \partial S$ by the open mapping theorem, and $f\left(z_{0}\right) \notin \mathbb{R}$.

And now, as the second main result of this section, we have

Theorem 3.7. If $|\beta|<1, P \in S(n, \beta)$ is 2-maximal with respect to $\alpha$ and $P^{\prime}(z) / \prod_{j=1}^{k}\left(z-z_{j}\right)^{m_{j}-1}$ has no multiple roots on the critical circle, then

(1) $2 r+s \geq k+2$ and

(2) $r \geq \frac{1}{6}(k+2)$.

Proof. By Theorem 2.21, the associated matrix must be positively singular, so there are numbers $\beta_{1}, \ldots, \beta_{r} \geq 0$ so that

$$
\sum_{i=1}^{r} \frac{\beta_{i} A\left(\zeta_{i}\right) z_{j}}{\left(\zeta_{i}-z_{j}\right)^{2}} \geq 0 \text { for } j=1, \ldots, s
$$

and

$$
\sum_{i=1}^{r} \frac{\beta_{i} A\left(\zeta_{i}\right)}{\left(\zeta_{i}-z_{j}\right)^{2}}=0 \text { for } j=s+1, \ldots, k
$$

Let $g(z)=\sum_{i=1}^{r} \beta_{i} A\left(\zeta_{i}\right) /\left(\zeta_{i}-z\right)^{2}$, then $z_{j} g\left(z_{j}\right) \geq 0$ for $j=1, \ldots, s$ and $g\left(z_{j}\right)=0$ for $j=s+1, \ldots, k$. Now $g(z)$ is a rational function with numerator of degree $\leq 2 r-2$, hence with at most $2 r-2$ zeros. Thus $k-s \leq 2 r-2$, so $2 r+s \geq k+2$. Further, $z g(z)$ is a rational function of maximal degree $2 r$ which is not real on all of $\{z:|z|=1\}$ (by Lemma 3.6, since $z g(z)$ is analytic on $\{z:|z| \geq 1\})$, so by Lemma $3.5 z g(z)$ is real on at most $4 r$ points of $\{z:|z|=1\}$, so $s \leq 4 r$. Then $r=\frac{1}{6}[(4 r-s)+(2 r+s)] \geq \frac{1}{6}(k+2)$.

\section{IMPLications OF CONJECTURE 2.5}

In this section, we examine some of the implications of Conjecture 2.5. Suppose the conjecture is true, suppose $|\beta|<1$ and suppose $P \in S(n, \beta)$ is monic and maximal with respect to $\alpha$. We may assume (by rotation) that $0 \leq \beta<1$. Since we are interested primarily in the cases $\alpha=\beta$ and $\alpha=\beta / 2$, we will suppose that $\alpha$ is also real. Our conjecture then implies that $P$ is a real polynomial. Write $P(z)=(z-\beta) Q(z)$, with $Q$ a real polynomial. Our conjecture implies that all roots of $P^{\prime}$ lie on the circle $\{z:|z-\alpha|=r\}$ (where $r=|P|_{\alpha}$ ), and that, given this, as many roots of $Q$ as possible lie on the circle $\{z:|z|=1\}$. Given this information, our goal is to compute $r$ as a function of $n, \alpha$, and $\beta$.

We will deal only with the case where all roots of $Q$ lie on the unit circle. As such, we assume the

Hypotheses 4.1. (1) $n \geq 2$ is an integer and $r, \alpha, \beta \in \mathbb{R}$ with $r>0$ and $0 \leq \beta<1$.

(2) $P(z)=(z-\beta) Q(z) \in S(n, \beta)$ is maximal with respect to $\alpha$. 
(3) All roots of $P^{\prime}$ lie on $\{z:|z-\alpha|=r\}$ (or equivalently, all roots of $P^{\prime}(r z+\alpha)$ lie on $\left.\{z:|z|=1\}\right)$.

(4) All roots of $Q$ lie on $\{z:|z|=1\}$.

To make use of this information, we will use the well-known

Lemma 4.2. If $\sum_{k=0}^{n-1} a_{k} z^{k}$ is a real polynomial of degree $n-1$ with all its roots on $\{z:|z|=1\}$ and with 1 as a root of even (resp. odd) multiplicity, then $a_{k}=a_{n-1-k}\left(\right.$ resp. $\left.a_{k}=-a_{n-1-k}\right)$ for $k=0, \ldots, n-1$.

This is particularly appropriate for our purposes, as we also have

Lemma 4.3. 1 is a root of $Q(z)$ of even multiplicity if and only if 1 is a root of $P^{\prime}(r z+\alpha)$ of even multiplicity.

Proof. Note first that $r>|\alpha-\beta|$ by the proof of Proposition 2.8, so $\alpha-r<$ $\beta<\alpha+r$. Since all roots of $P^{\prime}$ lie on $\{z:|z-\alpha|=r\}$, the only possible real root of $P^{\prime}$ greater than $\beta$ is $\alpha+r$.

If 1 is a root of $P^{\prime}(r z+\alpha)$ of even multiplicity, then $P^{\prime}$ has no real roots greater than $\beta$ of odd multiplicity. Since $\beta$ is a root of $P, P$ has no real roots greater than $\beta$, so 1 is not a root of $P$, i.e. 1 is a root of $Q$ of even (0) multiplicity.

If 1 is a root of $P^{\prime}(r z+\alpha)$ of odd multiplicity, then $P^{\prime}$ has 1 real root greater than $\beta$ of odd multiplicity. Since $\beta$ is a root of $P$, then $P$ has a real root greater than $\beta$. Since all roots of $Q$ lie on $\{z:|z|=1\}$, then 1 must be a root of $Q$. 1 cannot be a multiple root of $Q$ (else $P^{\prime}$ would have two distinct roots greater than $\beta$ ), so it must occur with odd (1) multiplicity.

Writing $Q(z)=\sum_{k=0}^{n-1} a_{k} z^{k}$, we have

$$
P(z)=(z-\beta) \sum_{k=0}^{n-1} a_{k} z^{k}=\sum_{k=-1}^{n-1}\left(a_{k}-\beta a_{k+1}\right) z^{k+1},
$$

so

$$
P^{\prime}(z)=\sum_{k=0}^{n-1}(k+1)\left(a_{k}-\beta a_{k+1}\right) z^{k}
$$

Then

$$
\begin{aligned}
P^{\prime}(r z+\alpha) & =\sum_{k=0}^{n-1}(k+1)\left(a_{k}-\beta a_{k+1}\right) \sum_{m=0}^{k}\left(\begin{array}{c}
k \\
m
\end{array}\right) \alpha^{k-m} r^{m} z^{m} \\
& =\sum_{m=0}^{n-1}\left[\sum_{k=m}^{n-1}(k+1)\left(a_{k}-\beta a_{k+1}\right)\left(\begin{array}{c}
k \\
m
\end{array}\right) \alpha^{k-m}\right] r^{m} z^{m}
\end{aligned}
$$


Now for $\alpha \neq 0$,

$$
\begin{aligned}
& \sum_{k=m}^{n-1}(k+1)\left(a_{k}-\beta a_{k+1}\right)\left(\begin{array}{c}
k \\
m
\end{array}\right) \alpha^{k-m} \\
& =(m+1) \sum_{k=m}^{n-1}\left(\begin{array}{c}
k+1 \\
m+1
\end{array}\right)\left(a_{k}-\beta a_{k+1}\right) \alpha^{k-m} \\
& =(m+1)\left[\sum_{k=m}^{n-1}\left(\begin{array}{c}
k+1 \\
m+1
\end{array}\right) a_{k} \alpha^{k-m}-\beta \sum_{k=m+1}^{n}\left(\begin{array}{c}
k \\
m+1
\end{array}\right) a_{k} \alpha^{k-m-1}\right] \\
& =(m+1) \sum_{k=m}^{n-1}\left[\alpha\left(\begin{array}{c}
k+1 \\
m+1
\end{array}\right)-\beta\left(\begin{array}{c}
k \\
m+1
\end{array}\right)\right] a_{k} \alpha^{k-m-1} \\
& =\sum_{k=m}^{n-1}\left(\begin{array}{c}
k \\
m
\end{array}\right)[\alpha(k+1)-\beta(k-m)] a_{k} \alpha^{k-m-1} .
\end{aligned}
$$

Define

$$
C_{m, k}= \begin{cases}\left(\begin{array}{l}
k \\
m
\end{array}\right)[\alpha(k+1)-\beta(k-m)] \alpha^{k-m-1} & \text { for } m<k \\
k+1 & \text { for } m=k \\
0 & \text { for } m>k\end{cases}
$$

Then for $\alpha \neq 0, C_{m, k}=\left(\begin{array}{l}k \\ m\end{array}\right)[\alpha(k+1)-\beta(k-m)] \alpha^{k-m-1}$ and so

$$
\sum_{k=m}^{n-1}(k+1)\left(a_{k}-\beta a_{k+1}\right)\left(\begin{array}{c}
k \\
m
\end{array}\right) \alpha^{k-m}=\sum_{k=0}^{n-1} C_{m, k} a_{k}
$$

Thus for $\alpha \neq 0, P^{\prime}(r z+\alpha)=\sum_{m=0}^{n-1}\left[\sum_{k=0}^{n-1} C_{m, k} a_{k} r^{m}\right] z^{m}$, and so by continuity

$$
P^{\prime}(r z+\alpha)=\sum_{m=0}^{n-1}\left[\sum_{k=0}^{n-1} C_{m, k} a_{k} r^{m}\right] z^{m}
$$

for all $\alpha$.

Recall that both $Q(z)$ and $P^{\prime}(r z+\alpha)$ have all their roots on $\{z:|z|=1\}$. Then Lemmas 4.2 and 4.3 give us that

$$
\begin{aligned}
\sum_{k=0}^{n-1} C_{m, k} a_{k} r^{m} & = \pm \sum_{k=0}^{n-1} C_{n-1-m, k} a_{k} r^{n-1-m} \\
& =\sum_{k=0}^{n-1} C_{n-1-m, n-1-k}\left( \pm a_{n-1-k}\right) r^{n-1-m} \\
& =\sum_{k=0}^{n-1} C_{n-1-m, n-1-k} a_{k} r^{n-1-m} .
\end{aligned}
$$


Thus for $n$ even and $m=0, \ldots,(n-2) / 2$, we have

$$
\begin{aligned}
0 & =\sum_{k=0}^{n-1} C_{n-1-m, n-1-k} r^{n-1-2 m} a_{k}-\sum_{k=0}^{n-1} C_{m, k} a_{k} \\
& =\sum_{k=0}^{(n-2) / 2} C_{n-1-m, n-1-k} r^{n-1-2 m} a_{k}-\sum_{k=0}^{(n-2) / 2} C_{m, k} a_{k}-\sum_{k=n / 2}^{n-1} C_{m, k} a_{k} \\
& =\sum_{k=0}^{(n-2) / 2} C_{n-1-m, n-1-k} r^{n-1-2 m} a_{k}-\sum_{k=0}^{(n-2) / 2} C_{m, k} a_{k}-\sum_{k=0}^{(n-2) / 2} C_{m, n-1-k}\left( \pm a_{k}\right) \\
& =\sum_{k=0}^{(n-2) / 2}\left(C_{n-1-m, n-1-k} r^{n-1-2 m}-C_{m, k} \mp C_{m, n-1-k}\right) a_{k} .
\end{aligned}
$$

This provides a system of $n / 2$ equations in the $n / 2$ "unknowns" $a_{0}, \ldots$, $a_{(n-2) / 2}$ which has a nontrivial solution (namely $\left.a_{0}, \ldots, a_{(n-2) / 2}\right)$, hence a zero determinant.

What we have shown is

Lemma 4.4. If $P$ satisfies Hypotheses 4.1 then the determinant of the matrix

$$
\left(\begin{array}{cclc}
C_{n-1, n-1} r^{n-1} & -C_{0,1} \mp C_{0, n-2} & \cdots & -C_{0,(n-2) / 2} \mp C_{0, n / 2} \\
-C_{0,0} \mp C_{0, n-1} & & & \\
C_{n-2, n-1} r^{n-3} \mp C_{1, n-1} & C_{n-2, n-2^{r}} r^{n-3} & \cdots & -C_{1,(n-2) / 2} \mp C_{1, n / 2} \\
\vdots & -C_{1,1} \mp C_{1, n-2} & & \vdots \\
C_{n / 2, n-1} r \mp C_{(n-2) / 2, n-1} & C_{n / 2, n-2} r \mp C_{(n-2) / 2, n-2} & \cdots & C_{n / 2, n / 2} r-C_{(n-2) / 2,(n-2) / 2} \\
& & & \mp C_{(n-2) / 2, n / 2}
\end{array}\right)
$$

is equal to 0 .

This determinant is a polynomial in $r$ of degree $(n-1)+(n-3)+\cdots+1=$ $(n / 2)^{2}$ with leading coefficient $\prod_{k=n / 2}^{n-1} C_{k k}=\prod_{k=n / 2}^{n-1}(k+1) \neq 0$.

We would now like to find the values of $r$ arising from particular choices of $n, \alpha$, and $\beta$. We restrict our search with

Lemma 4.5. Suppose that $P$ satisfies Hypotheses 4.1 .

Case 1. If $\alpha=\beta$ then $(1+\beta) / 2 \leq r \leq(1+\beta) / \sqrt[n-1]{n}$.

Case 2. If $\alpha=\beta / 2$ then $1 / 2 \leq r \leq \sqrt[n-1]{S(\beta) / n}$, where

$$
S(x)=2+(n-2)(1+x / 2)^{n-1}-(n-1)(1+x / 2)^{n-2} .
$$

Proof. Note that

$$
R(z)=\left(z-\frac{\beta-1}{2}\right)^{n}-\left(\frac{\beta+1}{2}\right)^{n} \in S(n, \beta) \quad \text { and } \quad|R|_{\alpha}=\left|\alpha-\frac{\beta-1}{2}\right| .
$$

Then for Case 1, $r \geq|R|_{\beta}=(1+\beta) / 2$ and for Case 2, $r \geq|R|_{\beta / 2}=1 / 2$.

Now write $P(z)=(z-\beta) Q(z)$, so

$$
n r^{n-1}=n \prod_{i=1}^{n-1}\left|\alpha-\zeta_{i}\right|=\left|P^{\prime}(\alpha)\right|=\left|(\alpha-\beta) Q^{\prime}(\alpha)+Q(\alpha)\right| .
$$


For Case 1,

$$
n r^{n-1}=|Q(\beta)|=\prod_{j=1}^{n-1}\left|\beta-z_{j}\right| \leq(1+\beta)^{n-1}
$$

so $r \leq(1+\beta) / \sqrt[n-1]{n}$. For Case 2 , define

$$
f(x)=\frac{x}{2} Q^{\prime}\left(\frac{x}{2}\right)-Q\left(\frac{x}{2}\right)
$$

and notice that $f^{\prime}(\beta)=\frac{\beta}{4} Q^{\prime \prime}\left(\frac{\beta}{2}\right)$ and

$$
\begin{aligned}
S^{\prime}(\beta) & =\frac{(n-1)(n-2)}{2}\left[\left(1+\frac{\beta}{2}\right)^{n-2}-\left(1+\frac{\beta}{2}\right)^{n-3}\right] \\
& =\frac{\beta}{4}(n-1)(n-2)\left(1+\frac{\beta}{2}\right)^{n-3} .
\end{aligned}
$$

Now $|f(0)|=|Q(0)|=1=S(0)$ and

$$
\left|f^{\prime}(\beta)\right|=\left|\frac{\beta}{4} Q^{\prime \prime}\left(\frac{\beta}{2}\right)\right| \leq \frac{\beta}{4}(n-1)(n-2)\left(1+\frac{\beta}{2}\right)^{n-3}=S^{\prime}(\beta),
$$

so

$$
n r^{n-1}=\left|\frac{\beta}{2} Q^{\prime}\left(\frac{\beta}{2}\right)-Q\left(\frac{\beta}{2}\right)\right|=|f(\beta)| \leq S(\beta),
$$

so $r \leq \sqrt[n-1]{S(\beta) / n}$.

Our search proceeds as follows: Given values for $\alpha$ and $\beta$ and the case ( $\alpha=\beta$ or $\alpha=\beta / 2$ ), we compute the bounds on $r$ as in Lemma 4.5. We then compute the coefficients of the polynomials given by the determinant in Lemma 4.4, use Sturm sequences to determine how many roots these polynomials have between our bounds, and find these roots. Starting with the largest of the roots and working downward, we compute the coefficients of $Q(z)$ and $P^{\prime}(r z+\alpha)$ and check to see if all their roots lie on the unit circle. The largest value of $r$ for which this occurs must give rise to a maximal polynomial (by Lemmas 4.4 and 4.5 , if there is a maximal polynomial $P$ which satisfies our hypotheses, then $r=|P|_{\alpha}$ must be among those roots).

The results of our search are summarized in Tables 4.6 and 4.7 and graphed in Figures 4.8 and 4.9. The curves terminate because there are some values of $\beta$ for which there are no polynomials satisfying Hypotheses 4.1.

Note especially the line $r=1-\beta / 2$ in Figure 4.9-this is the critical line for Conjecture 1.3. It is surpassed by the curves for $n=6,8,10$, and 12 , so Conjecture 1.3 is false for those values of $n$. Explicit counterexamples are as follows:

For $n=6$, let

$$
\begin{aligned}
Q_{6}(z)= & z^{5}+1.182303183 z^{4}+1.340070024 z^{3} \\
& +1.340070024 z^{2}+1.182303183 z+1
\end{aligned}
$$


TABLE 4.6. $r$ as a function of $n$ and $\beta$ for Case 1: $\alpha=\beta$.

\begin{tabular}{|c|c|c|c|c|c|}
\hline & $n=4$ & $n=6$ & $n=8$ & $n=10$ & $n=12$ \\
\hline .01 & .630766 & .699216 & .743277 & .774494 & .798000 \\
\hline .1 & .657715 & .719551 & .760376 & .789684 & .811920 \\
\hline .2 & .696206 & .752171 & .789374 & .816170 & .836526 \\
\hline .3 & .736324 & .786750 & .820252 & .844321 & .862540 \\
\hline .4 & .776559 & .821252 & .850777 & .871840 & .887662 \\
\hline$\beta \quad .5$ & .816332 & .854875 & .880062 & .897823 & * \\
\hline .6 & .855338 & .887202 & .907659 & $*$ & $*$ \\
\hline .7 & .893372 & .917976 & $*$ & $*$ & $*$ \\
\hline .8 & .930268 & * & $*$ & * & $*$ \\
\hline .9 & .965868 & * & $*$ & $*$ & $*$ \\
\hline .99 & .996658 & $*$ & $*$ & $*$ & * \\
\hline
\end{tabular}

TABLE 4.7. $r$ as a function of $n$ and $\beta$ for Case 2: $\alpha=\beta / 2$.

\begin{tabular}{|c|c|c|c|c|c|}
\hline & $n=4$ & $n=6$ & $n=8$ & $n=10$ & $n=12$ \\
\hline .01 & .629961 & .698827 & .742997 & .774263 & - \\
\hline .1 & .629439 & .698524 & .742761 & .774052 & .797595 \\
\hline .2 & .627049 & .696402 & .740806 & .772162 & .795719 \\
\hline .3 & .622339 & .691372 & .735777 & .767103 & .790592 \\
\hline .4 & .615076 & .682784 & .726790 & .757865 & .781126 \\
\hline .5 & .605034 & .670026 & .713013 & .743494 & .766299 \\
\hline .6 & .591940 & .652355 & .693405 & .722793 & .744826 \\
\hline .7 & .575446 & .628736 & .666447 & .693966 & * \\
\hline .8 & .555091 & .597597 & .629617 & $*$ & * \\
\hline .9 & .530241 & $*$ & $*$ & $*$ & * \\
\hline .99 & .503300 & $*$ & $*$ & * & - \\
\hline
\end{tabular}

A * means there is no polynomial which satisfies our hypotheses for those values of $n$ and $\beta$. A - means that no value could be computed with our algorithm. 
and $P_{6}(z)=(z-.84) Q_{6}(z)$. The roots of $Q_{6}$ are approximately $-1,-.506699$ $\pm .862122 i$ and $.415548 \pm .909571 i$ and they all lie on $\{z:|z|=1\}$, so $P \in S(6, .84)$. Conjecture 1.3 would require that at least one root of $P_{6}^{\prime}$ lies in $\{z:|z-.42| \leq .58\}$, but the roots of $P_{6}^{\prime}$ are approximately -.162507 , $-.159817 \pm .055911 i$ and $.098445 \pm .485712 i$, and they all lie on $\{z:|z-.42|=$ $.582507\}$, so Conjecture 1.3 is false when $n=6$.

For $n=8$, let

$$
\begin{aligned}
Q_{8}(z)= & z^{7}+1.241776468 z^{6}+1.504033112 z^{5}+1.702664563 z^{4} \\
& +1.702664563 z^{3}+1.504033112 z^{2}+1.241776468 z+1
\end{aligned}
$$

and $P_{8}(z)=(z-.8) Q_{8}(z)$. Then $P_{8} \in S(8, .8)$ but all roots of $P_{8}^{\prime}$ lie on $\{z:|z-.4|=.629617\}$, so Conjecture 1.3 is false when $n=8$.

For $n=10$, let

$$
\begin{aligned}
Q_{10}(z)= & z^{9}+1.401329769 z^{8}+1.873192265 z^{7}+2.303236288 z^{6} \\
& +2.551964562 z^{5}+2.551964562 z^{4}+2.303236288 z^{3} \\
& +1.873192265 z^{2}+1.401329769 z+1
\end{aligned}
$$

and $P_{10}(z)=(z-.7) Q_{10}(z)$. Then $P_{10} \in S(10, .7)$ but all roots of $P_{10}^{\prime}$ lie on $\{z:|z-.35|=.693967\}$, so Conjecture 1.3 is false when $n=10$.

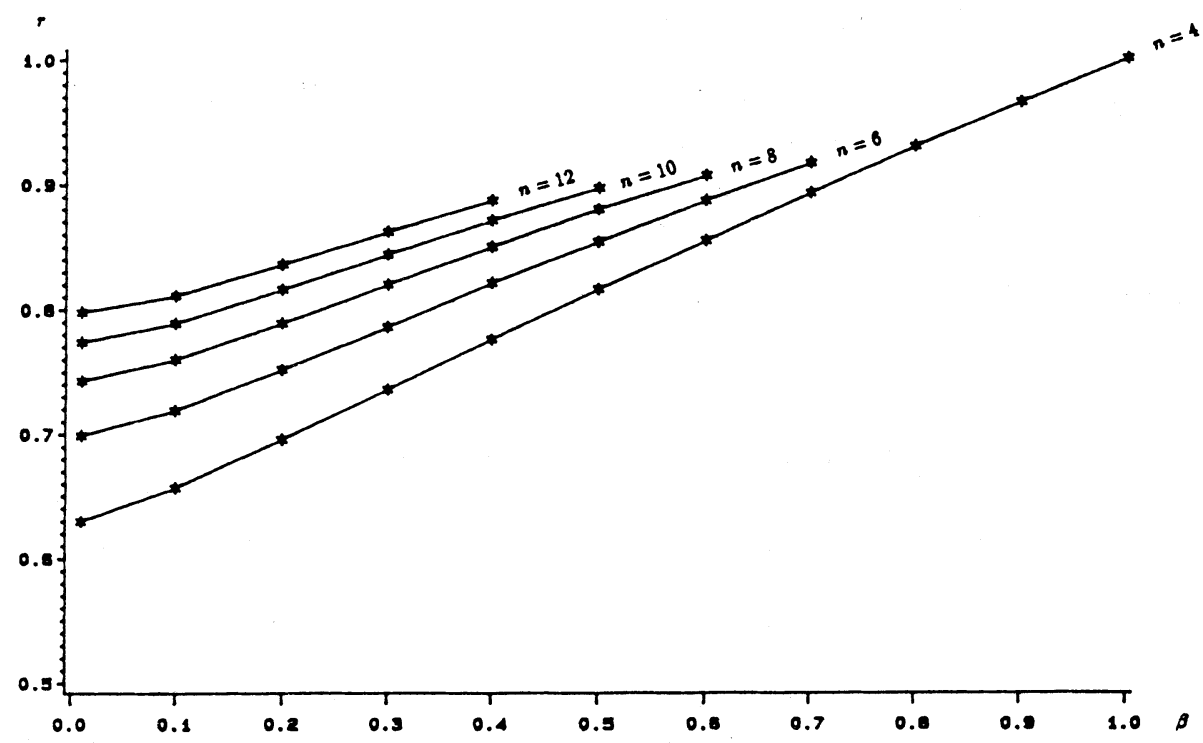

Figure 4.8. A graph of $r$ versus $\beta$ for Case 1: $\alpha=\beta$ 


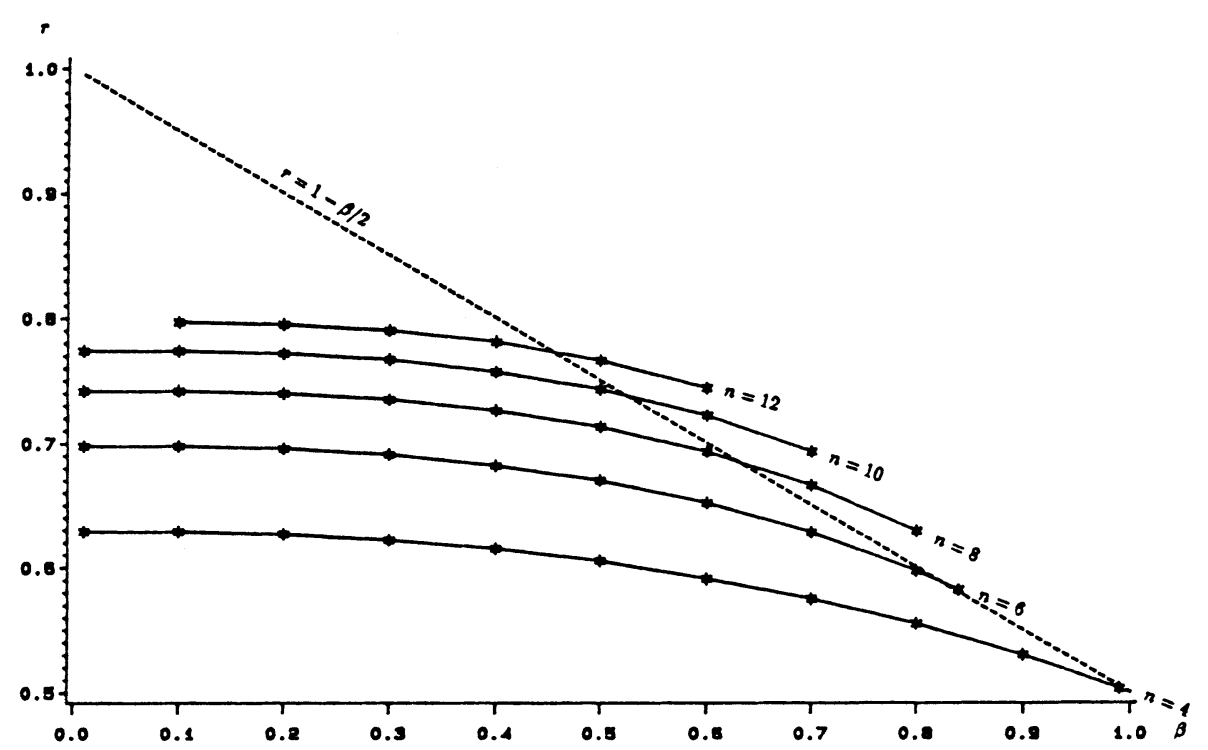

FIgURE 4.9. A graph of $r$ versus $\beta$ for Case 2: $\alpha=\beta / 2$

For $n=12$, let

$$
\begin{aligned}
Q_{12}(z)= & z^{11}+1.589848892 z^{10}+2.341118841 z^{9}+3.1133551299 z^{8} \\
& +3.725737921 z^{7}+4.054366178 z^{6}+4.054366178 z^{5} \\
& +3.725737921 z^{4}+3.113355299 z^{3} \\
& +2.341118841 z^{2}+1.589848892 z+1
\end{aligned}
$$

and $P_{12}(z)=(z-.6) Q_{12}(z)$. Then $P_{12} \in S(12, .6)$ but all roots of $P_{12}^{\prime}$ lie on $\{z:|z-.3|=.744827\}$, so Conjecture 1.3 is false when $n=12$.

Notice also that in Figure 4.9, for fixed $\beta, r$ appears to be an increasing function of $n$. For this reason, we make our

Conjecture 4.10. Conjecture 1.3 is false for all even $n \geq 6$.

\section{BIBLIOGRAPHY}

1. L. Ahlfors, Complex analysis, 2nd ed., McGraw-Hill, New York, 1966.

2. J. Farkas, Theorie der einfachen Ungleichungen, J. Reine Angew. Math. 124 (1901), 1-27.

3. F. Gacs, On polynomials whose zeros are in the unit disk, J. Math. Anal. Appl. 36 (1971), 627-637.

4. A. W. Goodman, Q. I. Rahman, and J. S. Ratti, On the zeros of a polynomial and its derivative, Proc. Amer. Math. Soc. 21 (1969), 273-274.

5. W. K. Hayman, Research problems in function theory, The Athelone Press, London, 1967.

6. F. Lucas, Theoremes concernant les equations algebriques, C. R. Acad. Sci. Paris 78 (1874), 431-433.

7. M. Marden, Geometry of polynomials, 2nd ed., Amer. Math. Soc., Providence, R. I., 1966. 
8. Conjectures on the critical points of a polynomial, Amer. Math. Monthly 90 (1983), 267-276.

9. A. Meier and A. Sharma, On Ilyeff's conjecture, Pacific J. Math. 31 (1969), 459-467.

10. D. Phelps and R. Rodriguez, Some properties of extremal polynomials for the Ilieff conjecture, Kodai Math. Sem Rep. 24 (1972), 172-175.

11. C. Pommerenke, Univalent functions, Vandenhoeck and Ruprecht, Göttingen, 1975.

12. G. Schmeisser, Bemerkungen zu einer Vermutung von Ilieff, Math. Z. 111 (1969), 121-125.

13. __ On Ilieff's conjecture, Math. Z. 156 (1977), 165-173.

Department of Mathematics, Le Moyne Colíege, Syracuse, New York 13214 